\title{
A Method Based on Combination Operation of Detecting Harmonic in Unbalanced Power System
}

\author{
Junli Wan, Hao Liu, Linjuan Wu, Shirun Cheng \\ College of Electrical Engineering \& New Energy, China Three Gorges University, Yi Chang, China
}

Email address:

wjlem@163.com (Junli Wan)

*Corresponding author

\section{To cite this article:}

Junli Wan, Hao Liu, Linjuan Wu, Shirun Cheng. A Method Based on Combination Operation of Detecting Harmonic in Unbalanced Power System. Science Journal of Energy Engineering. Vol. 4, No. 3, 2016, pp. 23-29. doi: 10.11648/j.sjee.20160403.11

Received: August 17, 2016; Accepted: August 26, 2016; Published: September 13, 2016

\begin{abstract}
Aiming at defects of traditional harmonic detection in three-phase unbalanced condition, an improved $i_{p}-i_{q}$ detecting method is proposed based on instantaneous reactive power theory. When power grid voltage unbalanced or voltage distortion, through coordinate transformation, trigonometric transforming and combination operation of the three-phase voltage, the fundamental positive sequence voltage is extracted, and the average filter string into the low pass filter (LPF) to reduce the ripples in fundamental positive sequence current. The improved method is more accurate and quick than traditional $i_{p}-i_{q}$ detecting method when extracting positive sequence fundamental voltage, detecting the negative sequence current of power grid and harmonic reactive current, significantly to reduce THD of fundamental positive sequence current and the detection error caused by unbalance and distortion voltage. Simulative results verify the correctness and validity of theoretical analysis.
\end{abstract}

Keywords: Instantaneous Reactive Power, Unbalanced Power Grid, Fundamental, Positive Sequence Voltage Extracting, Average Filter, Harmonic Detection

\section{Introduction}

With power electronic equipment as well as other non-linear loads widely being used in power systems, harmonic pollution problem arises making more serious impacting on power quality. Using active power filter (APF) to suppress the harmonic of power grid has become an important means of harmonic governance [1], meanwhile, harmonic detection of active power filter is the key to achieve accurate, real-time compensating current, also important for power system security, stability and economic operation. The mainly method of current harmonic detection including Fourier analysis and detection [2], Neural network adaptive testing [3], the instantaneous reactive power theory testing [4], etc. Based on Fast Fourier Transform (FFT) harmonic detection algorithm simply programming, easily implementing embedded system, however, it needs a certain period of time discrete sampling values when detecting. Harmonic detection algorithm based on nerve network features of speed, high accuracy, good real-time performance, capacity of study, real-time and anti-interference, nevertheless, there are still many problems in the practical application of nerve networks such as no standard method of neural network construction, no specific method for determining sample sizes and need a lot of training samples [5]. Harmonic detection algorithm based on nerve network is not mature, and further research needs to be optimized. Harmonic current detection based on instantaneous reactive power theory is widely used in harmonic detection since it has a small amount of calculation, simple model structure, and the advantages of good real-time performance [6-7]. The mainly method of detecting harmonic current based on instantaneous reactive power theory includes $p-q$ method and $i_{p}-i_{q}$ method. The $p-q$ method can only be used to detect the harmonic and reactive current of the three-phase voltage symmetrical system. Despite the $i_{p}-i_{q}$ method could detect harmonic when the system of three-phase voltage distortion and asymmetry, there is a large phase difference between the fundamental positive sequence voltage and the power grid voltage when power grid voltage distortion and imbalance, effecting the precision of phase locking and generating detection error [8], which cannot meet the requirements of APF precise compensation. In [9-10], the linear equations were constructed through the differential 
operation of three-phase voltage to separate fundamental positive sequence component of distortion power grid voltage, and then using phase locked loop (PLL) technique to compensate phase jump of power grid voltage to realize fast tracking of phase of fundamental positive sequence voltage component, but, when distorting voltage contain a lot of harmonic, could lead to dimension too many of the equations as well as complexity calculation, affecting dynamic response speed of the system. To slave the situation of slow response dynamic in single $d-q$ transforming, double synchronous transform phase locked loop and a decoupling network is adopted to extract positive sequence voltage component in imbalanced system [11-12], nevertheless, when harmonic contained in voltage, equipment cannot isolate fundamental component. The digital PLL of vary period sampling phase shift control loop is proposed by [13], the phase shifting controller is used to eliminate disturbance of the frequency doubling of the power grid, but it is unable to filter out other harmonic component. In [14], the improvement of the soft phase lock loop is improved by transfer filter function and reducing forward integral element, the dynamic phase tracking performance and unbalanced voltage detection performance are significantly improved. In [15], the basic principle of the non phase locked loop is analyzed and the average value theory is introduced, The method of the non phase locked loop harmonic detection based on the average value theory is proposed, but it needs to be transformed repeatedly and the computation is large. For harmonic detection error caused by traditional $i_{p}-i_{q}$ method in three-phase imbalanced, this paper, based on $i_{p}-i_{q}$ method, using virtual sine and cosine function fundamental positive sequence component of a phase voltage, existing in three-phase unbalance and the voltage distortion, are extracted by synchronizing the coordinate and trigonometric transformation; to eliminate the errors disturbing PLL detection by asymmetric and voltage distortion. Adding an average value filter to the Butterworth filter replace the traditional LPF obtaining current direct current component, to reduce the ripple current in the fundamental positive sequence.

\section{Traditional Harmonic Detection Algorithm Based on $i_{p}-i_{q}$ Theory and Reactive Power}

Based on instantaneous reactive power theory the traditional $i_{p}-i_{q}$ algorithm as in Fig. 1 [16-17], this method use the voltage of phase-A passing by the PLL getting sine signal in-phase of the voltage of phase-A and the corresponding cosine signal, and three-phase load current $i_{a}, i_{b}, i_{c}$ firstly obtain two-phase orthogonal current $i_{\alpha}, i_{\beta}$ by $\alpha-\beta$ transforming of the matrix $C_{32}$, as in (1), then obtaining active and reactive current $i_{p}, i_{q}$ by transformation of the matrix $C$, as in (2). And then getting the direct current component by LPF, finally getting the three-phase fundamental wave current $i_{a f}, i_{b f}, i_{c f}$ by reverse transformation of the matrix $C_{32}$, as in (3), and getting three-phase harmonic current $i_{a h}, i_{b h}, i_{c h}$ by $i_{a}, i_{b}, i_{c}$ subtracting fundamental of three-phase current respectively.

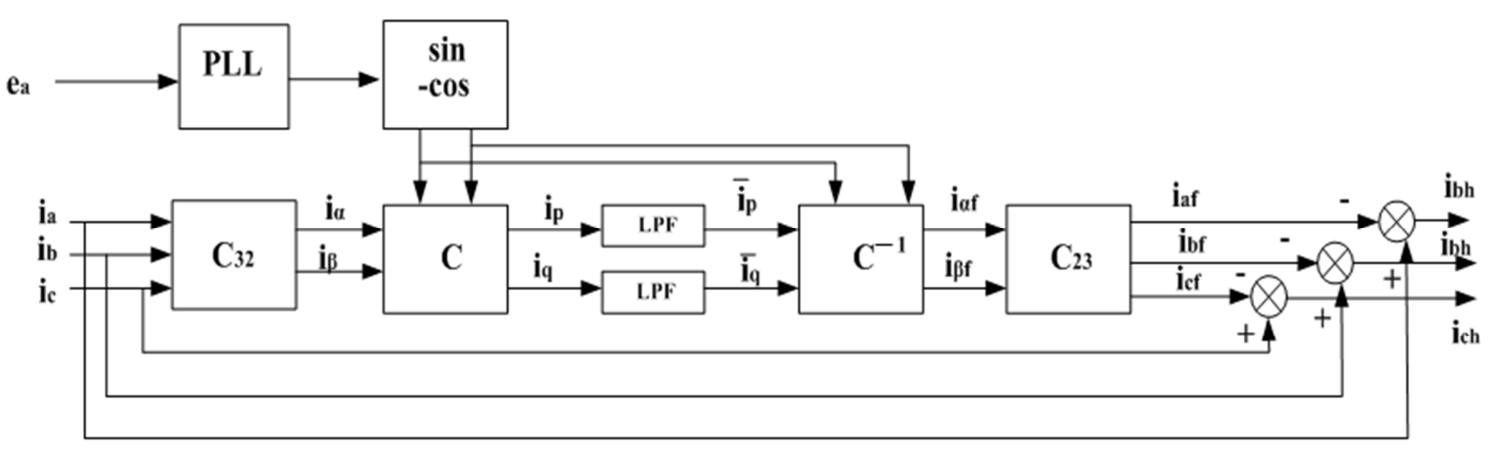

Fig. 1. The principle of harmonic detection based on ip - iq method.

$$
\begin{aligned}
& {\left[\begin{array}{c}
i_{\alpha} \\
i_{\beta}
\end{array}\right]=\sqrt{\frac{2}{3}}\left[\begin{array}{ccc}
1 & -\frac{1}{2} & 1 \\
0 & \frac{\sqrt{3}}{2} & -\frac{\sqrt{3}}{2}
\end{array}\right]\left[\begin{array}{l}
i_{a} \\
i_{b} \\
i_{c}
\end{array}\right]=C_{32}\left[\begin{array}{l}
i_{a} \\
i_{b} \\
i_{c}
\end{array}\right]} \\
& {\left[\begin{array}{c}
i_{p} \\
i_{q}
\end{array}\right]=\left[\begin{array}{cc}
\sin \omega t & -\cos \omega t \\
-\cos \omega t & -\sin \omega t
\end{array}\right]\left[\begin{array}{c}
i_{\alpha} \\
i_{\beta}
\end{array}\right]=C C_{32}\left[\begin{array}{c}
i_{a} \\
i_{b} \\
i_{c}
\end{array}\right]} \\
& {\left[\begin{array}{l}
i_{a f} \\
i_{b f} \\
i_{c f}
\end{array}\right]=\sqrt{\frac{2}{3}}\left[\begin{array}{cc}
1 & 0 \\
-\frac{1}{2} & \frac{\sqrt{3}}{2} \\
-\frac{1}{2} & -\frac{\sqrt{3}}{2}
\end{array}\right]\left[\begin{array}{cc}
\sin \omega t & -\cos \omega t \\
-\cos \omega t & -\sin \omega t
\end{array}\right]\left[\begin{array}{c}
\overline{i_{p}} \\
i_{q}
\end{array}\right]=C_{23} C^{-1}\left[\begin{array}{l}
\overline{i_{p}} \\
\overline{i_{q}}
\end{array}\right]}
\end{aligned}
$$

\section{The Modified Harmonic Detection Method}

When the asymmetry and distorted of three-phase voltage, the voltage of phase-A contains fundamental positive sequence voltage, negative sequence voltage, zero sequence voltage and harmonic voltage, making the phase difference between the voltage of phase-A and the positive sequence voltage of phase-A component. In the other hand, harmonic voltage will make great interference to the PLL, causing phase error of PLL tracking, ultimately affecting the harmonic and reactive current detection [18]; moreover, LPF of traditional $i_{p}$ $-i_{q}$ detection method makes large fundamental existing ripple 
because of the low order [19], which is more obvious when voltage imbalance and load unbalanced.

The principle of harmonic detection based on improved $i_{p}-i_{q}$ detection method as in Fig. 2, aiming at the characteristics of three-phase voltage imbalance and distortion, the synchronous rotating reference coordinate transformation and LPF being introduced before PLL to obtain the fundamental positive sequence voltage, and the LPF obtain direct current component has been improved at the same.

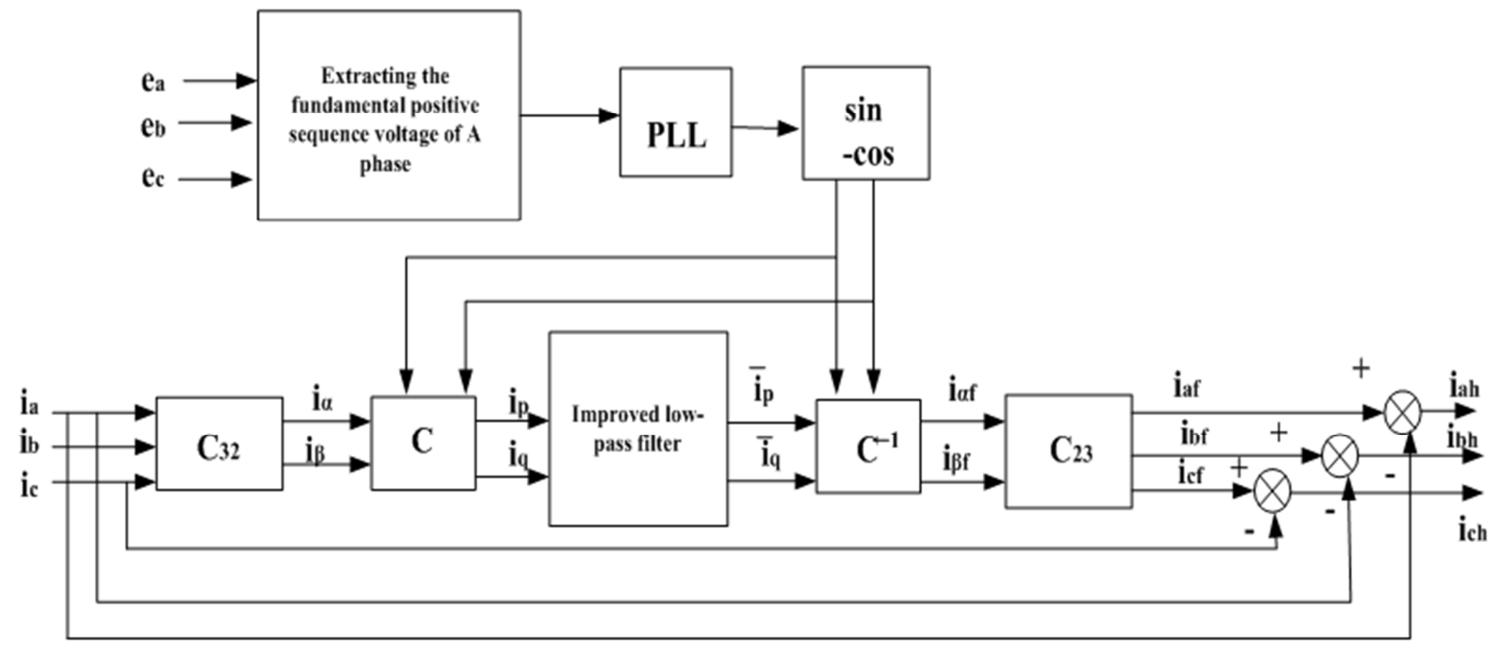

Fig. 2. The principle of improved ip - iq detection method.

\subsection{The Extraction of the Fundamental Positive Sequence Voltage of Phase-A}

The principle of extracting fundamental positive sequence voltage in phase-A as in Fig. 3, using virtual sine and cosine function, makes synchronous reference frame coordinate transforming, triangle function transforming and combination operation to eliminate direct current component of fundamental negative sequence voltage in phase-A. Furthermore, the fundamental positive sequence component is obtained by low pass filter. Ultimately, the fundamental positive sequence voltage in phase-A is constructed based on trigonometric.

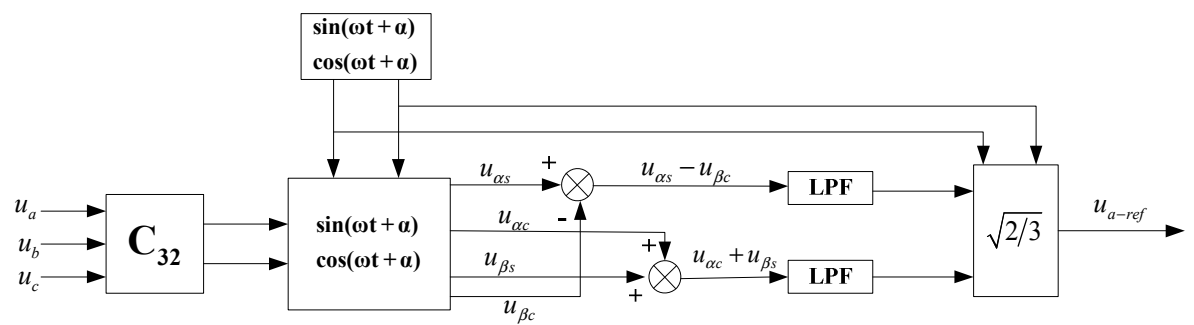

Fig. 3. The principle of extracting on fundamental positive sequence in a phase voltage.

If the three-phase grid voltage asymmetric containing harmonics as well, it can be expressed as:

$$
\left[\begin{array}{l}
u_{a} \\
u_{b} \\
u_{c}
\end{array}\right]=\left[\begin{array}{l}
\sqrt{2} \sum_{n=1}^{\infty}\left[U_{1 n} \sin \left(n \omega t+\varphi_{1 n}\right)+U_{2 n} \sin \left(n \omega t+\varphi_{2 n}\right)\right] \\
\sqrt{2} \sum_{n=1}^{\infty}\left[U_{1 n} \sin \left(n \omega t+\varphi_{1 n}-\frac{2 \pi}{3}\right)+U_{2 n} \sin \left(n \omega t+\varphi_{2 n}+\frac{2 \pi}{3}\right)\right. \\
\sqrt{2} \sum_{n=1}^{\infty}\left[U_{1 n} \sin \left(n \omega t+\varphi_{1 n}+\frac{2 \pi}{3}\right)+U_{2 n} \sin \left(n \omega t+\varphi_{2 n}-\frac{2 \pi}{3}\right)\right]
\end{array}\right]
$$

in (4), $\mathrm{U}_{1 \mathrm{n}}, \mathrm{U}_{2 \mathrm{n}}$ are respective positive and negative sequence component of valid values; $\varphi_{1 \mathrm{n}}, \varphi_{2 \mathrm{n}}$ are respective the initial phase of positive and negative sequence component; $\omega$ is the rotational angular frequency of grid voltage; $n$ is the order of harmonics. Transforming three-phase voltage into $\alpha$ $\beta$ coordinates it can be got that:

$$
\left[\begin{array}{l}
u_{\alpha} \\
u_{\beta}
\end{array}\right]=C_{32}\left[\begin{array}{l}
u_{a} \\
u_{b} \\
u_{c}
\end{array}\right]=\left[\begin{array}{l}
\sqrt{3} \sum_{n=1}^{\infty}\left[U_{1 n} \sin \left(n \omega t+\varphi_{1 n}\right)+U_{2 n} \sin \left(n \omega t+\varphi_{2 n}\right)\right] \\
\sqrt{3} \sum_{n=1}^{\infty}\left[-U_{1 n} \cos \left(n \omega t+\varphi_{1 n}\right)+U_{2 n} \cos \left(n \omega t+\varphi_{2 n}\right)\right]
\end{array}\right]
$$

Using (5) multiply unit sine and cosine matrix which are constructed it can be got that:

$$
\begin{gathered}
{\left[\begin{array}{l}
u_{\alpha s} \\
u_{\alpha c}
\end{array}\right]=u_{\alpha}\left[\begin{array}{l}
\sin (\omega t+\alpha) \\
\cos (\omega t+\alpha)
\end{array}\right]} \\
{\left[\begin{array}{l}
u_{\beta s} \\
u_{\beta c}
\end{array}\right]=u_{\beta}\left[\begin{array}{l}
\sin (\omega t+\alpha) \\
\cos (\omega t+\alpha)
\end{array}\right]}
\end{gathered}
$$

And properties of trigonometric function can be used to make combination operation among $u_{\alpha s}, u_{\alpha c}, u_{\beta s}, u_{\beta c}$, as in (8), fundamental negative sequence components are eliminated 
after being expanded. AC component is filtered by LPF, containing only fundamental positive sequence component value can be got as in (9).

$$
\begin{gathered}
{\left[\begin{array}{l}
u_{\alpha s}-u_{\beta c} \\
u_{\alpha c}+u_{\beta s}
\end{array}\right]=} \\
{\left[\begin{array}{c}
\sqrt{3} \sum_{n=1}^{\infty}\left[U_{1 n} \cos \left((n-1) \omega t+\varphi_{1 n}-\alpha\right)-U_{2 n} \cos \left((n+1) \omega t+\varphi_{2 n}+\alpha\right)\right] \\
\sqrt{3} \sum_{n=1}^{\infty}\left[U_{1 n} \sin \left((n-1) \omega t+\varphi_{1 n}-\alpha\right)-U_{2 n} \sin \left((n+1) \omega t+\varphi_{2 n}+\alpha\right)\right]
\end{array}\right]} \\
{\left[\begin{array}{c}
u_{\alpha s}-u_{\beta c} \\
u_{\alpha c}+u_{\beta s}
\end{array}\right]=\left[\begin{array}{l}
\sqrt{3} U_{11} \cos \left(\varphi_{11}-\alpha\right) \\
\sqrt{3} U_{11} \sin \left(\varphi_{11}-\alpha\right)
\end{array}\right]}
\end{gathered}
$$

Where, $U_{11}$ is the valid values of positive sequence component; $\varphi_{11}$ is the initial phase of positive sequence. By using change product into sum or difference of trigonometric function of trigonometric function, the constructed fundamental positive sequence in $a$ phase voltage $u_{\alpha-r e f}$ can be got [20], as in (10).

$$
\begin{aligned}
\mathrm{u}_{\alpha-\text {-ref }} & =\sqrt{\frac{2}{3}}\left[\left(\mathrm{u}_{\alpha \mathrm{s}}-\mathrm{u}_{\beta c}\right) \sin (\omega \mathrm{t}+\alpha)+\left(\mathrm{u}_{\alpha \mathrm{c}}+\mathrm{u}_{\beta \mathrm{s}}\right) \cos (\omega t+\alpha)\right] \\
& =\sqrt{2} \mathrm{U}_{11} \sin (\omega t+\alpha) \cos \left(\mathrm{j}_{11}-\alpha\right)+\sqrt{2} \mathrm{U}_{11} \cos (\omega t+\alpha) \sin \left(\mathrm{j}_{11}-\alpha\right) \\
& =\sqrt{2} \mathrm{U}_{11} \sin \left(\omega t+\mathrm{j}_{11}\right)
\end{aligned}
$$

The extractive fundamental positive sequence voltage of $a$ phase are being harmonic detected by phase-locked loop to eliminate the phase difference of $a$ phase voltage lock when voltage asymmetry and distortion, and avoid harmonic voltage signal interference on PLL.

\subsection{The Design of Improved Low Pass Filter}

In traditional $i_{p}-i_{q}$ detection method low pass filter (LPF) is used to obtain $i_{p}$ and $i_{q}$ direct current signal. When the higher the order, filter filtering effect is the better, but slower dynamic response. On the contrary, the lower the order of filter, the better of dynamic response but poor filtering effects nonetheless. When the order is same, the higher of cutoff frequency, the faster of dynamic response with low accuracy; the lower of cutoff frequency, the higher filtering precision with slower dynamic response. In a word, delay and filtering effect of the low pass filter is contradictory [21]. The advantage of filter based on Butterworth is balanced in three aspects: attenuation slope, linear phase and load characteristics [22]. Considered both the effect of filter and sensitive of response, the second-order Butterworth filter is selected and cut-off frequency regarded as $30 \mathrm{~Hz}$. This filter will bring a large ripple into direct component, especially when three-phase voltage unbalanced and load imbalance, which leads the fundamental positive sequence current containing higher harmonic, causing the harmonic detection resulting error.

The averaging filtering method uses module which calculates the averaging of $i_{p}$ and $i_{q}$ instead of LPF in $i_{p}-i_{q}$ algorithm. The mean current is a straight flow corresponded to current frequency component [23]. The principle of mean filtering as in Fig. 4:

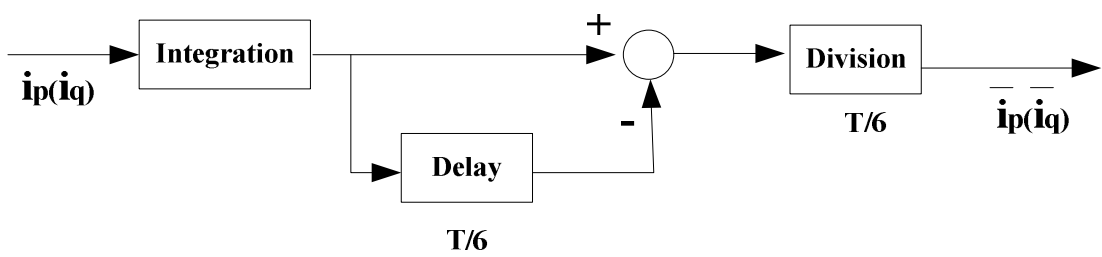

Fig. 4. Current mean filtering method.

Replace LPF with integral, time delay and gain link, algorithm implemented easily, and harmonic current detection delay is reduced to $\mathrm{T} / 6$. The averaging filtering method has faster dynamic response speed. When voltage stability and load symmetric, it has better real-time performance. But when the voltage distorted or load asymmetry, detection performance is relatively poor [24].

Considering the advantages and disadvantages of the above two kinds of filter algorithm, in this paper, use Butterworth filter and averaging filter which in series as a harmonic detection, in the form of modified LPF. The model diagram as in Fig. 5:

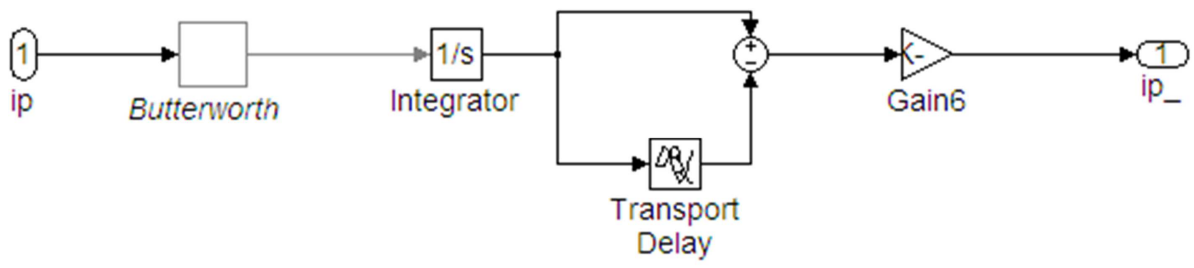

Fig. 5. Improved LPF based on harmonic detection method.

\section{Simulation Results and Analysis}

In order to verify the validity of the improved harmonic test, based on the above theoretical analysis and derivation, model and simulate of improved harmonic detection by matlab/simulink 
power system simulation software. Suppose three-phase power grids unbalanced, voltage of the simulation as in (11):

where, $\omega=2 \pi / f, f=50 \mathrm{~Hz}$. Three-phase voltage contains the fundamental positive sequence voltage and negative sequence voltage, 3rd and 5th harmonic voltage. Harmonic source is asymmetric load: three-phase controlled rectifier bridge and $\mathrm{A}$, $\mathrm{B}$ two-phase resistive load, DC side of the rectifier circuit is resistive inductive load $(\mathrm{R}=5 \mathrm{U}, \mathrm{L}=10 \mathrm{mH})$. The resistance of $\mathrm{A}$ and $\mathrm{B}$ is respective 5Üand $10 \mathrm{U}$.

Unbalanced three-phase power grid voltage simulation waveform is shown as in Fig. 6, and three-phase voltage unbalance and distortion; $a$ phase grid current and frequency spectrum analysis is shown as in Fig. 7. As can be seen that it contains a lot of harmonic current which is mainly 3rd, 5rd, $7 \mathrm{rd}$, 9rd harmonics, and total harmonic distortion (THD) is $35.42 \%$.

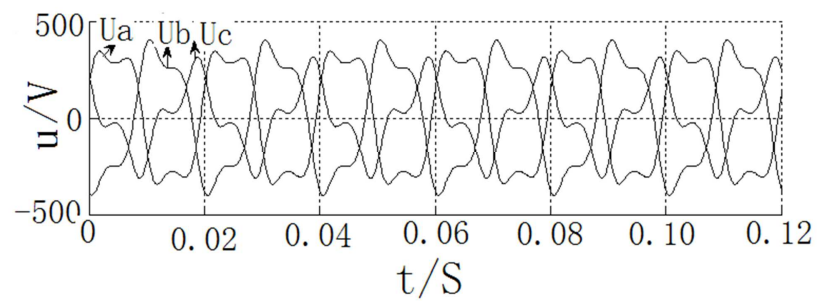

Fig. 6. Three-phase power grid voltage.

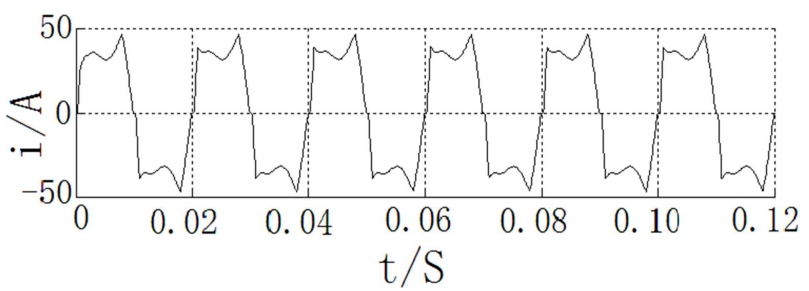

(a) Current of $a$ phase

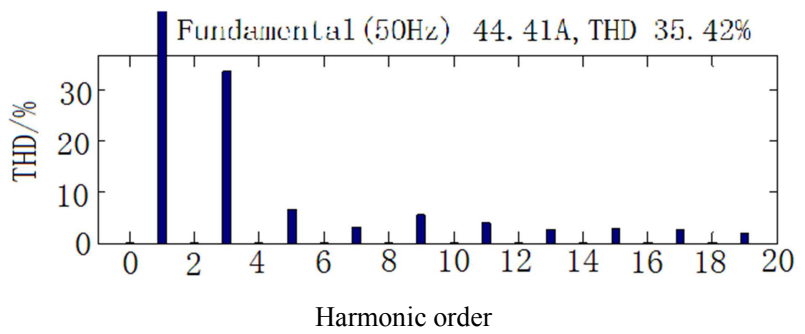

(b) frequency spectrum analysis of $a$ phase current

Fig. 7. Grid current of a phase.

Fig. 8 is simulation waveform got by traditional ip - iq method. Fig. 9 is simulation waveform got by improved ip - iq method.

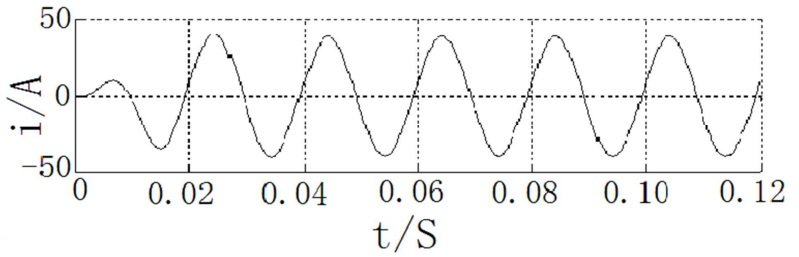

(a) Fundamental wave positive sequence active current of phase-A

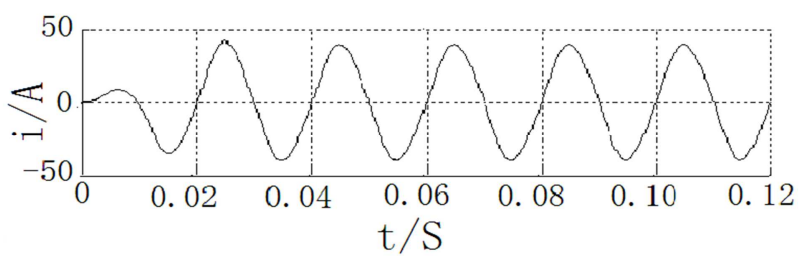

(b) Fundamental positive sequence active current of a phase by traditional $i_{p}$ $i_{q}$ method

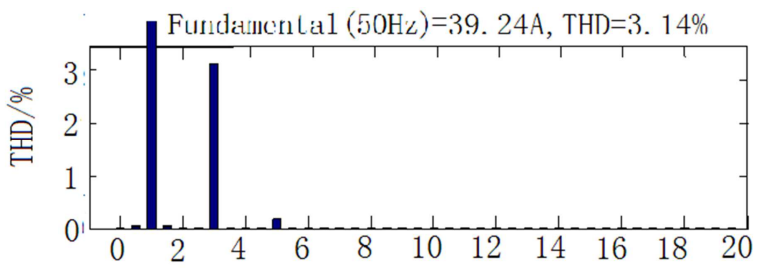

(c) Frequency spectrum analysis of $a$ fundamental positive sequence current

Fig. 8. Simulation waveforms by Traditional $i_{p}-i_{q}$ method.

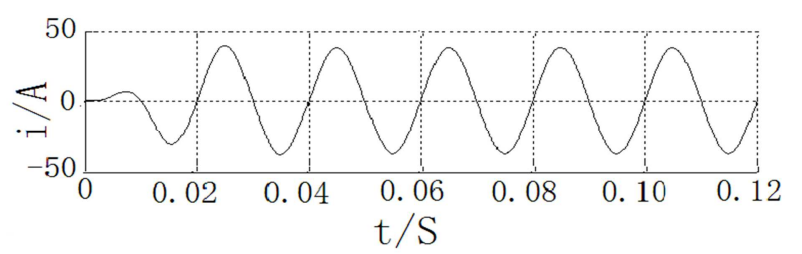

(a) Fundamental wave positive sequence active current of $a$ phase

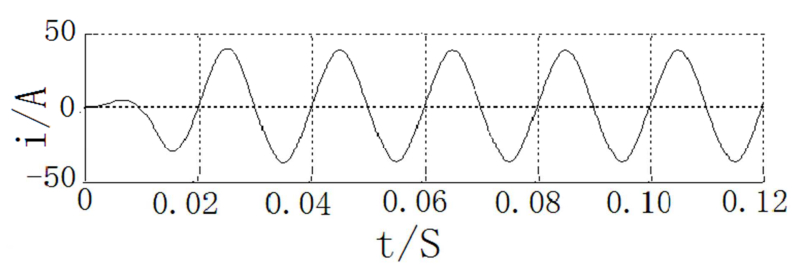

(b) Fundamental positive sequence active current of a phase

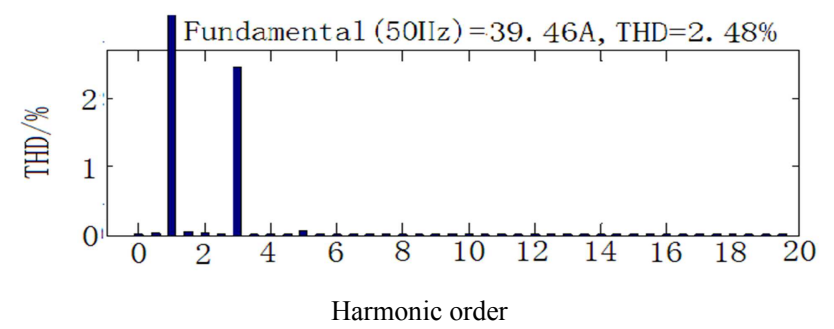

(c) Frequency spectrum analysis of $a$ fundamental positive sequence current 


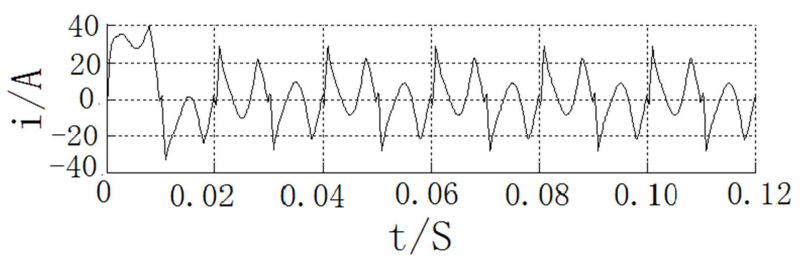

(d) The sum of negative sequence current, harmonic current and reactive current

Fig. 9. Simulation waveforms by improved $i_{p}-i_{q}$ method.

Comparing Fig. 8 (a) with Fig. 9 (a), it can be got that the phase of fundamental positive sequence active current by traditional $i_{p}-i_{q}$ method has a deviation. The initial phase is not zero simultaneously with the positive sequence voltage, and also has a deviation when compared with improved $i_{p}-i_{q}$ method of the amplitude of fundamental positive sequence active current which will lead to big error when detecting reactive current. Improved $i_{p}-i_{q}$ method, whereas, can accurately obtains the fundamental positive sequence active current when three-phase voltage asymmetry and distorted. Comparing Fig. 8 (c) with Fig. 9 (c), it can be got that the fundamental positive current of $a$ phase by improved $i_{p}-i_{q}$ method has large of ripple. Comparing of the frequency analysis results of fundamental positive sequence current of $a$ phase by this two methods, it can be got that THD of fundamental positive sequence current got by traditional $i_{p}-i_{q}$ method is $3.14 \%$, while THD of fundamental positive sequence current after improving is $2.48 \%$. According to the results, it can be known that improved $i_{p}-i_{q}$ method keeps better dynamic response speed when three-phase imbalance, and it can accurately detects the sum of negative sequence current, reactive current and harmonic current.

\section{Conclusion}

This paper based on the theory of instantaneous reactive power, analyses the harmonic detection results got by $i_{p}-i_{q}$ method when three-phase imbalance, and an improved $i_{p}-i_{q}$ method is put forward. The improved $i_{p}-i_{q}$ method has simple circuits and computation, and more accurately and quickly to extract the positive sequence fundamental voltage, detecting the negative sequence current of power grid, harmonic and reactive current when three-phase imbalance compared with traditional method. It can eliminate the error caused by imbalance and distorted voltage, and improved LPF in the link of current filter is used to reduce ripple in the positive sequence, the detection accuracy improved at the same time. Simulation results show the effectiveness of harmonic detection by improved $i_{p}-i_{q}$ method when three-phase system of power grid unbalanced.

\section{Acknowledgement}

This work was supported by the Natural Science Foundation of Hubei Province, China (No. 2010CDB10803).

\section{References}

[1] Huang ZC, Su XP, Li J (2015) Asymmetrical three-phase system harmonic detection and compensation method research. Power Electronic Technology 49 (3): 82-84 (in Chinese).

[2] Xue H, Yang RG, (2002) High precision harmonic detection algorithm based on FFT. Proceedings of the CSEE 22 (12): 106-110 (in Chinese).

[3] Li ZC, Ren MW, Zhu DH et al (2011). An adaptive detecting algorithm for harmonic current. Power Electronics 45 (7): 50-51.

[4] Zhou L, Meng Q, Liu Q et al (2009). Detection method based on instantaneous reactive power theory of harmonic and reactive current. High Voltage Technique 30 (2): 385-392 (in Chinese).

[5] Rao Y, Zheng JW (2006) A kind of fundamental wave extraction based on steepest descent algorithm of adaptive filter Electric Power Automation Equipment 26 (8): 19-22 (in Chinese).

[6] He YJ, Liu JJ, Wang ZA, Zou YP (2010) A digital harmonic detection based on instantaneous reactive power theory. Transactions of China Electrotechnical Society 25 (8): 185-192 (in Chinese).

[7] Zhang XG, Xu D, Li WW (2009) A digital phase locking loop applying to unbalance situation of the grid. Electro technical Application 28 (6): 54-58.

[8] Zhou HL, Wan JR, Li SC et al (2012) Voltage distortion and imbalance state without phase-locked loop UPQC offsets detection method. Electric Power Automation Equipment 32 (5): 50-56 (in Chinese).

[9] Naidu SR, Mascarenhas AW, Fernandes DA (2004). A software phase-locked loop for unbalanced and distorted utility conditions. In: Proceedings of the 2004 IEEE International Conference on Power System Technology, Singapore, Nov 2004, 999-1004.

[10] Zhou P, He YK (2010) Synchronization signal detection of fundamental voltage under harmonic distorted grid voltage conditions. Chinese Journal of Scientific Instrument 31 (1): 78-84. (in Chinese).

[11] Rodriguez P, Pou J, Ergas J et al (2007) Decoupled double synch-ronous reference frame PLL for power converters control. IEEE Trans on Power Electronics 22 (2): 584-592.

[12] Tian GZ, Tu ST, Lin BJ et al (2010) Design of phase locked loop based on double synchronous reference frame under unbalance grid voltage in wind power systems. Electric Drive 40 (7): 53-57.

[13] Zhang XG, Xu DG, Li WW (2009) A kind of applicable to the power grid unbalance fault condition of digital phase-locked loop. Electrotechnical Application 28 (6): 54-58 (in Chinese).

[14] Yuan ZC, Sung Q, Liu WH (2010) Improving the dynamic phase tracking and unbalanced voltage detection performance improvements soft phase locked loop algorithms. Power System Technology 34 (1): 31-35. 
[15] Ding XJ, Zhang HJ, Zhang XF (2010) A harmonics-detection method without PLL and based on average theory in three-phase ciruit. Electrical Measurement \& Insrumentation 47 (553): 43-47 (in Chinese).

[16] Xu Z (2009) The instantaneous reactive power theory and its application in electric power regulation. China Machine Press, Beijing (in Chinese).

[17] Ma YC (2009) Harmonic detection method based on instantaneous reactive power research. Mechanical and electrical integration technology at home and abroad 1 (3): 50-52 (in Chinese).

[18] Han S, Qiu GY, Peng XJ (2005) Effect of phase difference on Harmonic and Reactive Current Detection Analysis. Electrical Measurement \& Instrumentation (4): 1-3 (in Chinese).

[19] Zhou K, Luo A, Xia XY el at (2007) An Improved ip-iq Harmonic Detecting Method and Digital Low Pass Filter Optimized Design. Proceedings of the CSEE 27 (34): 96-101.

\section{Biography}

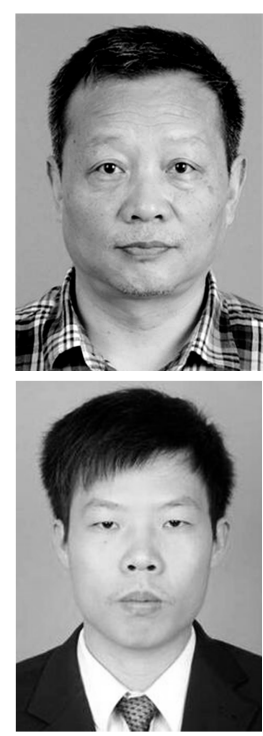

Junli Wan was born in 1957. Now he is a professor and Master supervisor in the College of Electrical Engineering \& New Energy, China Three Gorges University. His present research interests include Smart Grid Technologies, intelligent information processing technology and its application in power systems.

Hao Liu was born in 1990. Now he is a student in the College of Electrical Engineering \& New Energy, China Three Gorges University. He present research interests are about the smart grid and power quality.
[20] Zhang XL, Liu MG, Zhong HZ (2014) $p$ - $q$ harmonic detection algorithm and simulation analysis. Electrical Measurement \& Instrumentation 17: 24-28 (in Chinese).

[21] Ye HW (2011) The research of current predictive control of active power filter. Dissertation, University of Xi'an Jiaotong University (in Chinese).

[22] Zhang YQ, Song WL, Gao JX (2010) Advanced low pass filter in the application of variable harmonic source load harmonic detection. Journal of Electric Power 25 (6): 475-479 (in Chinese).

[23] Tang Z, Chen YW, Liao DF el at (2009) Optimized low-pass filter harmonic detection circuit parameters. Shanghai Electric Power Univercity Journal (4): 374-378 (in Chinese).

[24] Hai-ya JIANG, Wen-juan LI, Jian-hua DU (2010) Comparative analysis of three fundamental active power filter extraction methods 29 (1): 97-100 (in Chinese).

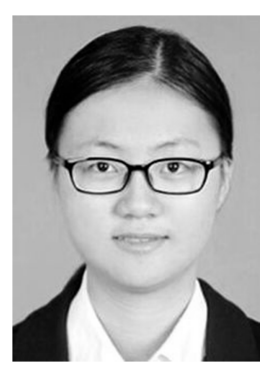

Linjuan Wu was born in 1994. Now she is a student in the College of Electrical Engineering \& New Energy, China Three Gorges University. Her research interests are about the smart grid and power quality.

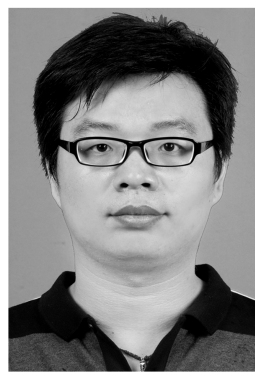

Shirun Cheng was born in 1989. Now he is a student in the College of Electrical Engineering \& New Energy, China Three Gorges University. He present research interests are about the smart grid and intelligent control. 